PITHA 99/17

hep-ph/9906399

\title{
Direct determination of the gluon density in the proton from jet cross sections in deep-inelastic scattering
}

\author{
M. Wobisch \\ III. Physikalisches Institut, RWTH Aachen, \\ D-52056 Aachen, Germany
}

\begin{abstract}
A QCD analysis of jet production in deep-inelastic scattering is presented. The studies are based on recent measurements of jet cross sections in the H1 experiment at HERA which are directly sensitive to the gluon density in the proton. The inclusion of $\mathrm{H} 1$ data on the inclusive deep-inelastic scattering cross section enables us to perform a consistent, simultaneous determination of the quark and gluon densities in the proton. The gluon density has been determined at fairly large values of $10^{-2}<x<10^{-1}$ at a scale of the order of the transverse jet energies $\mu_{f}^{2}=200 \mathrm{GeV}^{2}$. The result is consistent with indirect determinations from HERA structure function data and extends their range of sensitivity to larger $x$-values.
\end{abstract}

Talk given on behalf of the H1 collaboration at the 7th International Workshop on Deep-Inelastic Scattering and QCD (DIS99), Zeuthen, April 1999. 


\title{
Direct determination of the gluon density in the proton from jet cross sections in deep-inelastic scattering
}

\author{
M. Wobisch ${ }^{\mathrm{a}}$ for the H1 collaboration \\ aIII. Physikalisches Institut, RWTH Aachen, D-52056 Aachen, Germany
}

A QCD analysis of jet production in deep-inelastic scattering is presented. The studies are based on recent measurements of jet cross sections in the H1 experiment at HERA which are directly sensitive to the gluon density in the proton. The inclusion of $\mathrm{H} 1$ data on the inclusive deep-inelastic scattering cross section enables us to perform a consistent, simultaneous determination of the quark and gluon densities in the proton. The gluon density has been determined at fairly large values of $10^{-2}<x<10^{-1}$ at a scale of the order of the transverse jet energies $\mu_{f}^{2}=200 \mathrm{GeV}^{2}$. The result is consistent with indirect determinations from HERA structure function data and extends their range of sensitivity to larger $x$-values.

\section{INTRODUCTION}

The present knowledge on the gluon density in the proton basically comes from deep-inelastic scattering (DIS) structure function data (i.e. from inclusive DIS cross sections). These are, however, only indirectly sensitive to the gluon density, which enters the cross section formulae only via the next-to-leading order (NLO) corrections in the boson-gluon fusion process.

A process that is directly sensitive to the gluon density in the proton is the production of jets at (moderately) high transverse energies $\left(E_{T}\right)$ in the Breit frame. At leading order, high $E_{T}$ jet cross sections are described by QCD-Compton and by boson-gluon fusion processes, the latter being dominant over large regions of phase space. A QCD analysis of jet cross sections may therefore lead to a direct determination of the gluon density, independent of assumptions needed in the indirect determinations from structure function data.

\section{EXPERIMENTAL RESULTS}

The dijet and the inclusive jet cross sections presented here have been measured with the H1 detector at HERA, based on data taken in the years 1994-97 corresponding to an integrated luminosity $\mathcal{L}_{\text {int }} \simeq 36 \mathrm{pb}^{-1}$. The kinematic range extends from moderate to large momentum trans- fers $10<Q^{2}<5000 \mathrm{GeV}^{2}$ for $0.2<y<0.6$. Jets are defined by the inclusive $k_{\perp}$ algorithm [ 1 which is applied to the final state particles in the Breit frame. Only jets in the central region of the detector acceptance with pseudorapidities $-1<\eta_{\text {jet,lab }}<2.5$ and transverse jet energies $E_{\text {TBreit }}>5 \mathrm{GeV}$ are considered.

The inclusive jet cross section $\mathrm{d}^{2} \sigma_{\text {jet }} / \mathrm{d} E_{T} \mathrm{~d} Q^{2}$ has been measured for $7<E_{T \text { jet,Breit }}<50 \mathrm{GeV}$. For the dijet cross section events with two or more jets have been selected where the two jets with the highest $E_{T}$ fulfill $\sum E_{T}>17 \mathrm{GeV}$. Double differential distributions have been obtained for a large set of variables [2]. Here we show the dependence of the inclusive jet cross section on both hardness scales $E_{T}, Q^{2}$ (Fig. 1), and the dependence of the dijet cross section on the jet pseudorapidity $\eta^{\prime}$ and the reconstructed fractional parton momentum $\xi$ (Figs. 2.3). The basic observations are: towards larger $Q^{2}$ a harder $E_{T}$ spectrum is seen; large jet pseudorapidities $\eta^{\prime}$ are suppressed at higher $E_{T}$; the jet cross sections are sensitive to fractional parton momenta $0.005<\xi<0.3$.

The perturbative calculations give a very good description of these and other distributions [ 2], except at small values of $Q^{2}<100 \mathrm{GeV}^{2}$ where NLO corrections are very large (k-factors are between 1.6 and 2) and sizeable contributions from higher orders are expected. 


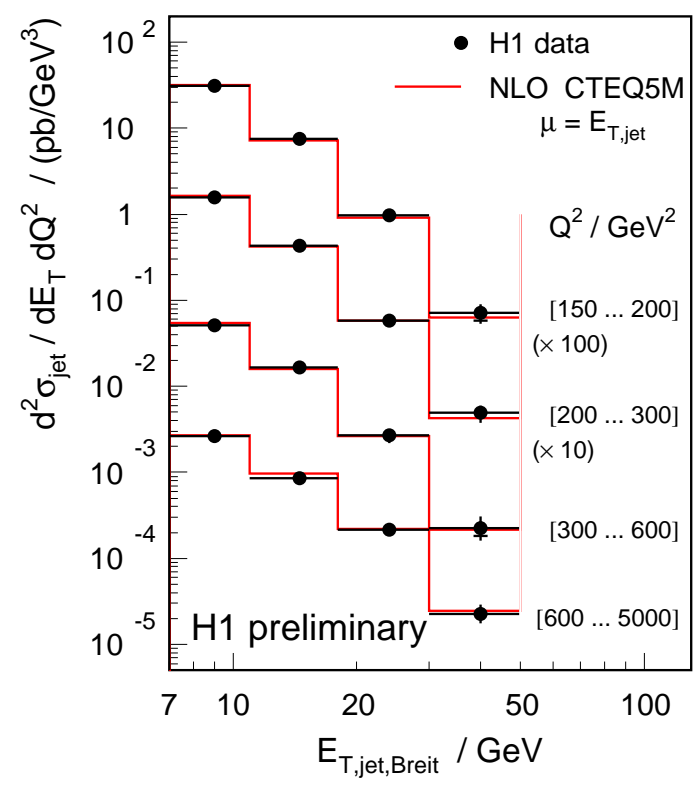

Figure 1. The inclusive jet cross section as a function of the transverse jet energy $E_{T}$ in the Breit frame in different regions of $Q^{2}$.

\section{QCD ANALYSIS}

We have seen that the jet data are nicely described by perturbative calculations at NLO when parton densities and $\alpha_{s}\left(M_{Z}\right)$ values are taken from global fits. We therefore conclude that perturbative QCD at next-to-leading order $\alpha_{s}$ is able to describe jet production processes in DIS, at least in the kinematic region under investigation: at fairly large transverse jet energies $E_{T}$ in the Breit frame and momentum transfers $Q^{2}$ not too small. The influence of non-perturbative contributions has been investigated [3] and is found to be small (below $7 \%$ for the dijet cross sections) with negligible model dependence.

It is now straightforward to make a QCD analysis of these data for a determination of the free parameters of the theory: $\alpha_{s}$, the gluon density and the quark densities in the proton. The present data are, however, not able to constrain these simultaneously. We therefore decided to take the value of $\alpha_{s}\left(M_{Z}\right)$ (as e.g. measured by the LEP experiments independently of the proton structure)

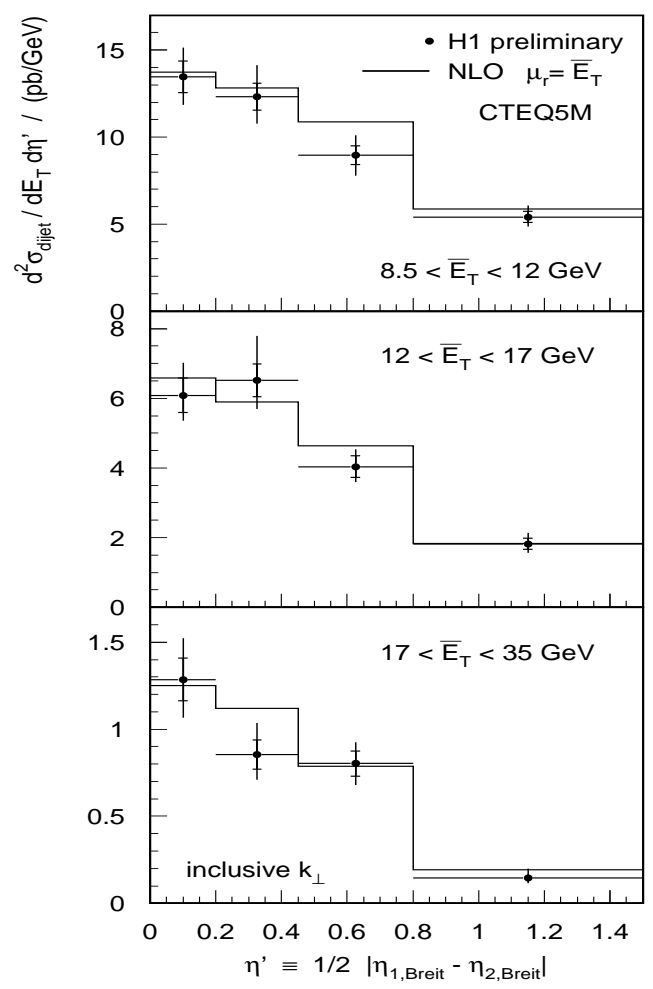

Figure 2. The dijet cross section in different regions of the average transverse jet energy $\bar{E}_{T}$ in the Breit frame as a function of the jet pseudorapidity $\eta^{\prime}$ (reconstructed from the pseudorapidity difference of the jets in the Breit frame $\left.\eta^{\prime}=1 / 2\left|\eta_{1}-\eta_{2}\right|\right)$. The measurement is performed at $200<Q^{2}<5000 \mathrm{GeV}^{2}$.

as external input, and perform a simultaneous fit of the gluon and quark densities in the proton. For this purpose we include H1 data on the inclusive DIS cross section [ [4] in the QCD analysis at $Q^{2}$ values which are of the order of the transverse jet energies $E_{T}^{2}$ in the dijet cross sections.

In this kinematic region $\left(200<Q^{2}<\right.$ $650 \mathrm{GeV}^{2}$ ) the inclusive DIS cross sections give very strong constraints on the quark densities while they depend on the gluon density only weakly. In the combined fit of both datasets the inclusive DIS cross sections therefore determine the quarks while the dijet cross sections determine the gluon density. 


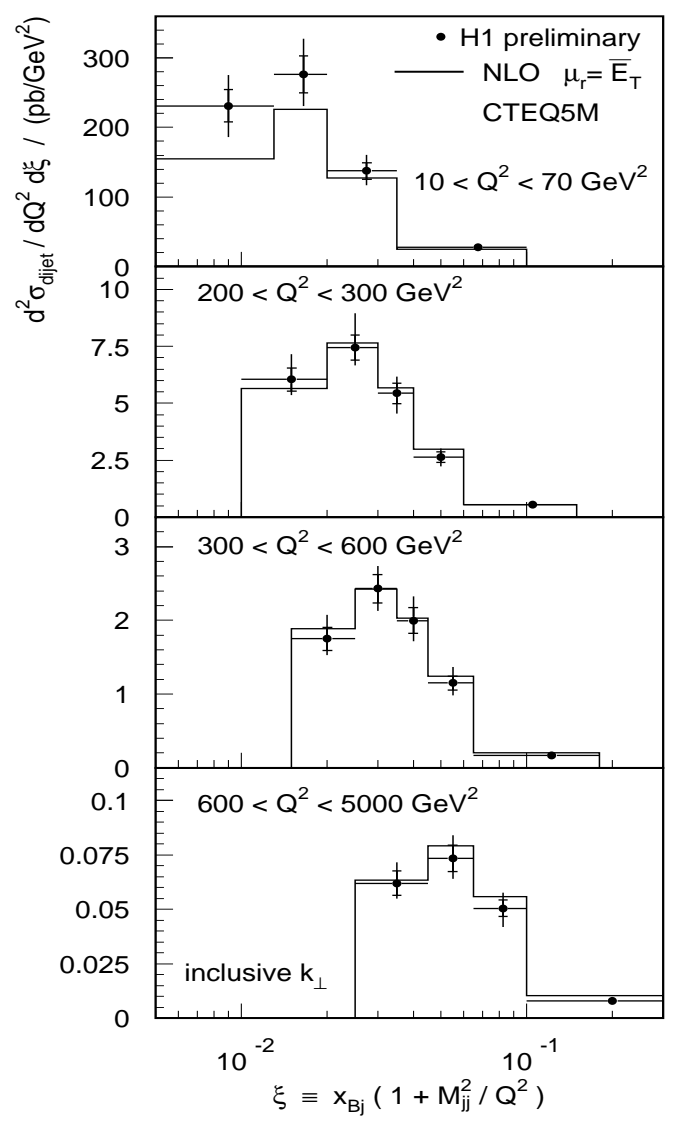

Figure 3. The dijet cross section in different regions of $Q^{2}$ as a function of the fractional parton momentum $\xi$ reconstructed from the invariant mass of the dijet system.

The dijet data used in the fit are restricted to $Q^{2}>200 \mathrm{GeV}^{2}$ where NLO corrections and hadronization corrections are small. The fit has been performed to the double differential cross sections $\mathrm{d}^{2} \sigma_{\text {dijet }} / \mathrm{d} Q^{2} \mathrm{~d} \xi, \quad \mathrm{d}^{2} \sigma_{\text {dijet }} / \mathrm{d} Q^{2} \mathrm{~d} x_{\mathrm{Bj}}$ and $\mathrm{d}^{2} \sigma_{\text {incl. }} / \mathrm{d} Q^{2} \mathrm{~d} x_{\mathrm{Bj}}$ which are most sensitive to the $x$-dependence of the parton distributions. The gluon and the quark densities have been fitted at a factorization scale $\mu_{f}^{2}=200 \mathrm{GeV}^{2}$ which is of the size of the hard scales for both the dijet $\left(\mu_{f}^{2} \simeq E_{T}^{2}\right)$ and the inclusive DIS cross sections $\left(\mu_{f}^{2} \simeq Q^{2}\right)$. More details on the fitting procedure can be found in [2].

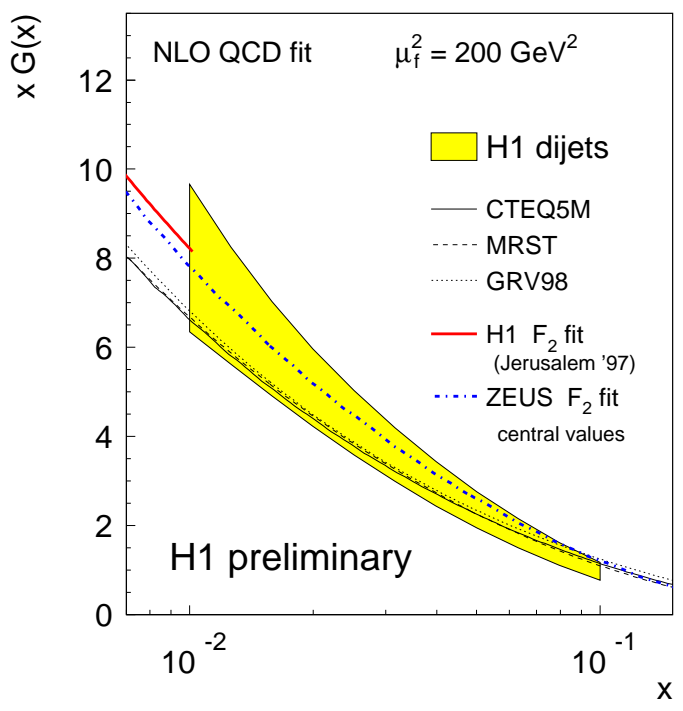

Figure 4. The gluon density in the proton in the $\overline{\mathrm{MS}}$-scheme determined in a NLO QCD fit to dijet cross sections at a factorization scale $\mu_{f}^{2}=$ $200 \mathrm{GeV}^{2}$ for a value of $\alpha_{s}\left(M_{Z}\right)=0.119 \pm 0.005$. The error band is compared to results from global fits and to the results of QCD fits to HERA structure function data.

The resulting gluon density is displayed in Fig. 专 at the scale $\mu_{f}^{2}=200 \mathrm{GeV}^{2}$ in the range $0.01<x<0.1$. The error band includes experimental and theoretical uncertainties as well as the uncertainty of $\alpha_{s}\left(M_{Z}\right)$. Within these uncertainties this direct determination is consistent to the results from global fits and to indirect determinations from HERA structure function data and extends their range of sensitivity to larger $x$-values.

\section{REFERENCES}

1. S. D. Ellis, D. E. Soper, Phys. Rev. D48 (1993) 3160.

2. H1 Collaboration, contributed paper 520 to ICHEP 98, Vancouver, Canada (1998).

3. M. Wobisch, T. Wengler, to appear in proceedings of "Monte-Carlo Generators for HERA Physics", Hamburg (1999).

4. H1 Collaboration, contributed paper 533 to ICHEP 98, Vancouver, Canada (1998). 